\title{
Checklist of parasites in fish from the upper Paraná River floodplain: An update
}

\author{
Lista de parasitos em peixes da planície de inundação do alto rio Paraná: \\ uma atualização
}

\begin{abstract}
Atsler Luana Lehun ${ }^{1 *}$ (1); Wagner Toshio Hasuike2; João Otávio Santos Silva1; Juliana Rosa Matias Ciccheto³ Gabriela Michelan'; Aparecida de Fátima Cracco Rodrigues²; Danilo Nunes Nicola²; Lucas Duarte de Lima²; Amanda Nardi Correia2; Ricardo Massato Takemoto ${ }^{1,2,4}$

'Programa de Pós-graduação em Ecologia de Ambientes Aquáticos Continentais, Universidade Estadual de Maringá - UEM, Maringá, PR, Brasil 2 Programa de Pós-graduação em Biologia Comparada, Universidade Estadual de Maringá - UEM, Maringá, PR, Brasil

${ }^{3}$ Programa de Pós-graduação em Ecologia e Conservação, Universidade Federal do Paraná - UFPR, Curitiba, PR, Brasil. ${ }^{4}$ Núcleo de Pesquisas em Limnologia, Ictiologia e Aquicultura - Nupélia, Universidade Estadual de Maringá - UEM, Maringá, PR, Brasil
\end{abstract}

How to cite: Lehun AL, Hasuike WT, Silva JOS, Ciccheto JRM, Michelan G, Rodrigues AFC, et al. Checklist of parasites in fish from the upper Paraná River floodplain: An update. Braz J Vet Parasito/ 2020; 29(3): e008720. https://doi.org/10.1590/S1984-29612020066

\begin{abstract}
This study provides an updated list of parasite species and their respective hosts in the upper Paraná River floodplain. The list of parasites is structured by phylum, class, order and family, followed by a record of each host species. A total of 315 taxa of parasites were reported, of which 201 were identified at the species level. These 201 species comprise 3 Flagellata, 3 Myxozoa, 50 Monogenea, 43 Digenea, 40 Cestoda, 41 Nematoda, 8 Acanthocephala, 6 Copepoda, 5 Branchiura and 2 Pentastomida, arranged in 84 host fish species. This work carried out in the floodplain of the upper Paraná River contributes to the listing of parasite species and host interactions of the local ichthyofauna. Little is known about these communities and its riches are underestimated due to the high density of fish found in this region, highlighting the importance of conducting studies on the local fauna.
\end{abstract}

Keywords: Monogenea, Digenea, Cestoda, Nematoda, Acanthocephala, Crustacea.

\section{Resumo}

Este estudo fornece uma lista atualizada de espécies de parasitos e seus respectivos hospedeiros da planície de inundação do alto rio Paraná. A lista de parasitos está estruturada em filo, classe, ordem e família, seguida do registro de cada espécie de hospedeiro. São relatados 315 táxons de parasitos e, destes, 201 foram identificados por espécie. Essas 201 espécies compreendem: 3 Flagellata, 3 Myxozoa, 50 Monogenea, 43 Digenea, 40 Cestoda, 41 Nematoda, 8 Acanthocephala, 6 Copepoda, 5 Branchiura e 2 Pentastomida, distribuídos em 84 espécies de hospedeiros. Este trabalho, realizado na planície de inundação do alto rio Paraná, contribui para a listagem de espécies de parasitos e interações parasito-hospedeiro da ictiofauna local. Pouco dessas comunidades é conhecida, e suas riquezas são subestimadas devido à alta densidade de peixes encontrados nessa região, destacando-se a importância da realização de estudos sobre a fauna local.

Palavras-chave: Monogenea, Digenea, Cestoda, Nematoda, Acanthocephala, Crustacea.

\section{Introduction}

The Paraná River is the tenth longest river in the world in water discharge and it is located in south-central South America, running through Brazil, Paraguay and Argentina. The upper Paraná River floodplain is the only dam-free stretch remaining, located between the Porto Primavera and Itaipu dams (Agostinho et al., 2001). In this stretch, the river has a vast portion of wide-braided channels from numerous lagoons, whether permanent or temporary, which are distributed throughout the plain and are fed by groundwater or by the flooding process (Junk et al., 1989; Agostinho \& Júlio, 1999; Thomaz et al., 2004). 
The recurrent floods experienced by this ecosystem are an important selection pressure that favors certain adaptations in the organisms inhabiting this area, resulting in communities with unique structures and functions (Junk et al., 1989; Thomaz et al., 2007). One of the main characteristics of floodplain river systems in tropical and subtropical regions is the high diversity of species, particularly of fish (Horne \& Goldman, 1994; Toussaint et al., 2016; Vitule et al., 2017). In this context, the high diversity of the environment can alter the population dynamics of the ichthyofauna, along with their ecological, physiological and biological conditions. This, in turn, influences the structure and composition of parasitic fauna in fish (Pavanelli et al., 1997; Yamada et al., 2008; Luque \& Poulin, 2008; Locke et al., 2014), including the trophic category and the intermediate hosts of parasites (Landsberg et al., 1998).

The parasite fauna of fish from the upper Paraná River floodplain were previously detailed by Pavanelli et al. (1997), Takemoto et al. (2009) and further studies that reported taxonomic descriptions of new species, such as Ferrari-Hoeinghaus et al. (2010), Tavernari et al. (2010), Karling et al. (2011, 2014). Over the last decade, studies recorded in this area have indicated an increase in the diversity of fish parasites, so the purpose of this study was to provide an updated list of parasites and their respective fish hosts in the upper Paraná River floodplain.

\section{Material and Methods}

This study was conducted with data from published articles, dissertations, thesis, and research project reports (consult supplementary material) on parasites from fish carried only at the Laboratório de Ictioparasitologia of Universidade Estadual de Maringá between 1988 and 2019, in the region of the upper Paraná River floodplain (Figure 1).

The list of parasite species presented in this study is clustered by phylum and classified in class, order, and family. For Myxozoa and Flagellata, the classification is in accordance with Brusca et al. (2018a,b); for Monogenea,

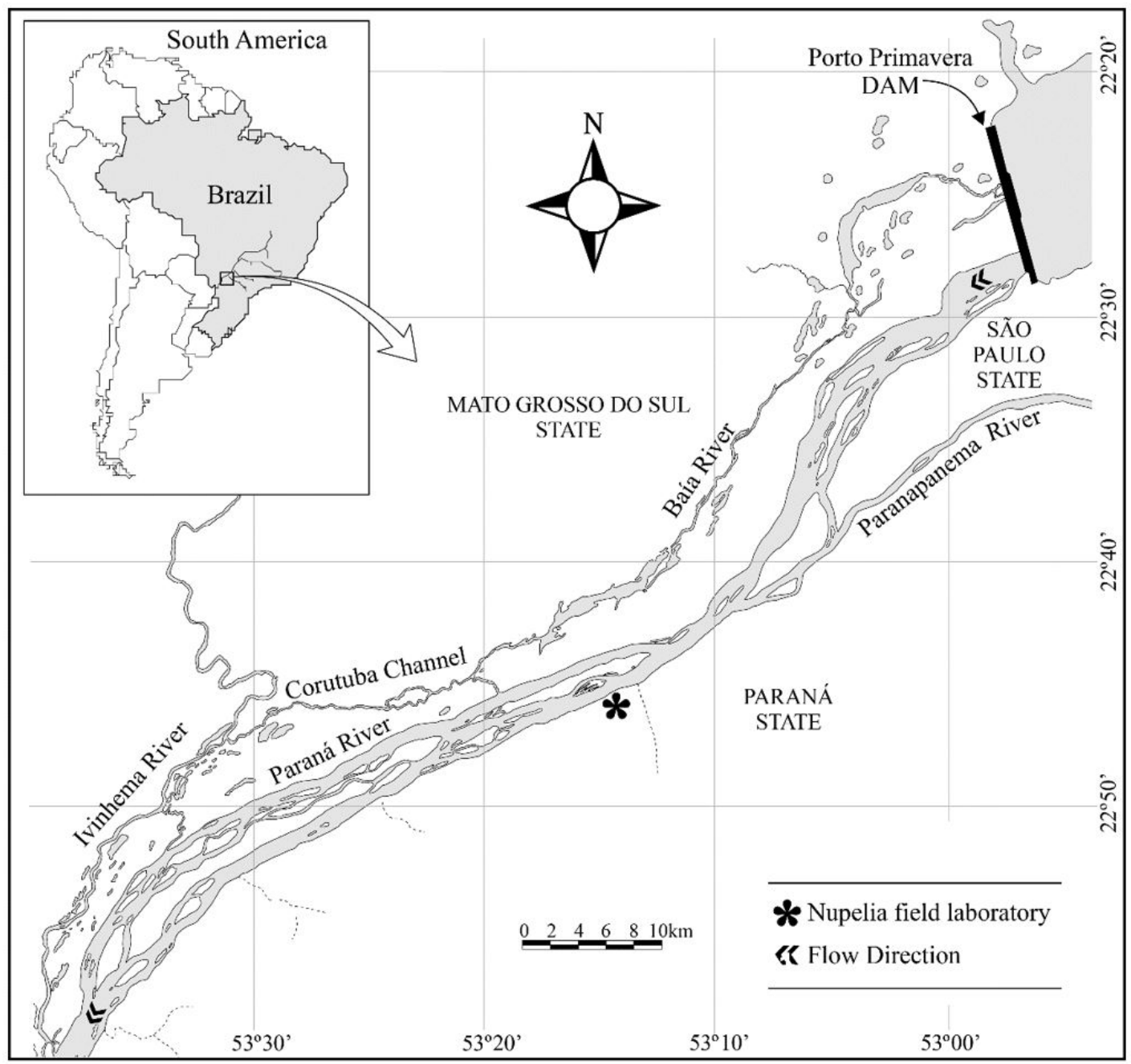

Figure 1. The upper Paraná River floodplain. From Jaime Luiz Lopes Pereira. 
Cohen et al. (2013); for Digenea, Thatcher (1993) and Kohn et al. (2007); for Cestoda, Khalil et al. (1994) and VidalMartínez et al. (2000); for Nematoda, Moravec (1998); for Acanthocephala, Yamaguti (1959) and Thatcher (2006); for Crustacea, Eiras et al. (2010), Ahyong et al. (2011) and Luque et al. (2013); and for Hirudinea and Acari, Brusca et al. $(2018 c, d)$. Some informative changes in recent literature, such as the World Register of Marine Species, have also been considered. When the species are unknown, the parasites were cataloged in class, family or genus, while the identified species are presented in alphabetical order, followed by the author and year of publication. For the hosts, the identification and registration of occurrence in the floodplain were checked and confirmed according to Ota et al. (2018), and these are listed opposite their respective parasites. Information about the parasite's life stage and the infection site was also added.

For the quantification of interactions, all association formed between a given parasite and its host was considered. For parasites with more than one host, the number of interactions was added, taking into account all their respective hosts. We consider the most specific taxonomic level of the parasite, since all hosts are at the species level.

\section{Results}

In this study, 13 groups of parasites were registered for 84 species of ichthyofauna from the upper Paraná River floodplain, of which eight were groups of ectoparasites and six of endoparasites, with emphasis on the Pentastomida group considered for two types of parasitism. Three hundred and fifteen taxa were cataloged and 201 of these were identified at the species level. These 201 species comprise 3 Flagellata, 3 Myxozoa, 50 Monogenea, 43 Digenea, 40 Cestoda, 41 Nematoda, 8 Acanthocephala, 6 Copepoda, 5 Branchiura, and 2 Pentastomida. The parasites belonging to Isopoda, Hirudinea and Acarina were identified only at the group level, but are added together in the interaction records.

The parasites of the Monogenea class had the largest number of identified species, totaling 50. However, the largest number of different interactions recorded, regardless of the level of identification (order, family, or larval stage, immature form), were the parasites of the phylum Nematoda with 227 interactions, followed by the Digenea subclass with 176 interactions, and then the Monogenea class with 149 interactions.

\section{Parasite-Host List}

\section{FLAGELLATA}

Phylum Euglenozoa Cavalier-Smith, 1981

Class Kinetoplastea Honigberg, 1963

Order Trypanosomatida (Kent, 1880)

Family Trypanosomatidae Doflein, 1901

Trypanosoma guairaensis Eiras, Rego \& Pavanelli, 1989 - Megalancistrus parananus (Peters, 1881), blood. Trypanosoma nupelianus Eiras, Rego \& Pavanelli, 1990 - Rhinelepis aspera Spix \& Agassiz, 1829, blood. Trypanosoma scrofae Eiras \& Pavanelli, 1989 - Prochilodus lineatus (Valenciennes, 1836), blood.

\section{MYXOZOA}

Phylum Cnidaria Hatschek, 1888

Class Myxozoa Grassé, 1970

Order Bivalvulida Shulman, 1959

Family Myxobolidae Thélohan, 1892

Henneguya caudicula Eiras, Takemoto \& Pavanelli, 2008 - Leporinus lacustris Campos, 1945, spore, gills. Henneguya corruscans Eiras, Takemoto \& Pavanelli, 2009 - Pseudoplatystoma corruscans (Spix \& Agassiz, 1829), spore, gills. Henneguya paranaensis Eiras, Pavanelli \& Takemoto, 2004 - Prochilodus lineatus, spore, gills.

\section{MONOGENEA}

Phylum Platyhelminthes Minot, 1876

Class Monogenea (Van Beneden, 1858)

Monogenea fam. gen. sp. - Acestrorhynchus lacustris Lütken, 1875, Aphyocharax anisitsi Eigenmann \& Kennedy, 1903, Auchenipterus osteomystax (Miranda Ribeiro, 1918), Crenicichla sp., Geophagus brasiliensis (Quoy \& Gaimard, 
1824), Hypophthalmus oremaculatus Nani \& Fuster, 1947, Hypostomus regani (Ihering, 1905), Hypostomus ternetzi (Boulenger, 1895), Iheringichthys labrosus (Lütken, 1874), Leporellus vittatus Valenciennes, 1850, Loricaria sp., Loricariichthys platymetopon Isbrücker \& Nijssen, 1979, Parauchenipterus galeatus (Linnaeus, 1766), Piaractus mesopotamicus (Holmberg, 1887), Pimelodus maculatus (Lacépède, 1803), Plagioscion squamosissimus (Heckel, 1840), Potamotrygon amandae Loboda \& Carvalho, 2013, Pseudoplatystoma corruscans, Rhamdia quelen (Quoy \& Gaimard, 1824), Rhaphiodon vulpinus Spix \& Agassiz, 1829, Rhinelepis aspera, Roeboides descalvadensis Fowler, 1932, Salminus brasiliensis (Cuvier, 1816), Satanoperca sp., Schizodon nasutus Kner, 1858, Serrasalmus marginatus Valenciennes, 1837, Trachydoras paraguayensis (Eigenmann \& Ward, 1907), gills.

\section{Order Dactylogyridea Bychowsky, 1937 Family Dactylogyridae Bychowsky, 1933}

Ameloblastella paranaensis (França, Isaac, Pavanelli \& Takemoto, 2003) - Iheringichthys labrosus, gills.

Amphithecium sp. - Astyanax lacustris (Lütken, 1875), Schizodon borellii (Boulenger, 1900), Serrasalmus marginatus, gills. Amphocleithrium sp. - Pseudoplatystoma corruscans, gills.

Amphocleithrium paraguayensis Price \& Romero, 1969 - Pseudoplatystoma corruscans, gills.

Anacanthocotyle anacanthocotyle Kritsky \& Fritts, 1970 - Astyanax lacustris, Moenkhausia forestii Benine, Mariguela \& Oliveira, 2009, gills.

Anacanthoroides sp. - Prochilodus lineatus, gills.

Anacanthorus sp. - Erythrinus erythrinus (Bloch \& Schneider, 1801), Hoplerythrinus unitaeniatus (Agassiz, 1829), Hoplias spp., Metynnis lippincottianus (Cope, 1870), Salminus brasiliensis, Serrasalmus maculatus Kner, 1858, Serrasalmus marginatus, gills.

Anacanthorus bicuspidatus Cohen, Kohn \& Boeger, 2012 - Salminus brasiliensis, gills.

Anacanthorus contortus Cohen, Kohn \& Boeger, 2012 - Salminus brasiliensis, gills.

Anacanthorus douradensis Cohen, Kohn \& Boeger, 2012 - Salminus brasiliensis, gills.

Anacanthorus parakruidenieri Cohen, Kohn \& Boeger, 2012 - Salminus brasiliensis, gills.

Anacanthorus penilabiatus Boeger, Husak \& Martins, 1995 - Piaractus mesopotamicus, gills.

Anacanthorus toledoensis Cohen, São Clemente \& Leão, 2015 - Piaractus mesopotamicus, gills.

Ancyrocephalinae gen. sp. - Prochilodus lineatus, Pseudoplatystoma corruscans, Schizodon borellii, Serrasalmus marginatus, gills.

Annulotrematoides sp. - Schizodon borellii, gills.

Apedunculata sp. - Schizodon borellii, gills.

Characithecium costaricensis (Price \& Bussing, 1967) - Moenkhausia forestii, gills.

Cleidodiscus sp. - Laetacara araguaiae Ottoni \& Costa, 2009, Leporinus friderici Bloch, 1794, Leporinus lacustris, Megaleporinus piavussu (Britski, Birindelli \& Garavello, 2012), Satanoperca sp., gills.

Cosmetocleithrum bulbocirrus Kritsky, Thatcher \& Boeger, 1986 - Hoplias spp., gills.

Dactylogyridae gen. sp. - Hoplias spp., gills.

Demidospermus sp. - Auchenipterus osteomystax, Pimelodus maculatus, Serrasalmus marginatus, gills.

Demidospermus labrosi Monteiro, Kritsky \& Brasil-Sato, 2010 - Iheringichthys labrosus, gills.

Demidospermus osteomystax Tavernari, Takemoto, Lacerda \& Pavanelli, 2010 - Auchenipterus osteomystax, gills.

Demidospermus paranaensis Ferrari-Hoeinghaus, Bellay, Takemoto \& Pavanelli, 2010 - Loricariichthys platymetopon, gills.

Diplectanum piscinarius Kritsky \& Thatcher, 1984 - Plagioscion squamosissimus, gills.

Gussevia sp. - Astronotus crassipinnis (Heckel, 1840), gills.

Gussevia arilla Kritsky, Thatcher \& Boeger, 1986 - Cichla kelberi Kullander \& Ferreira, 2006, gills.

Gussevia asota Kritsky, Thatcher \& Boeger, 1989 - Astronotus crassipinnis, gills.

Gussevia astronoti Kritsky, Thatcher \& Boeger, 1989 - Astronotus crassipinnis, gills.

Gussevia longihaptor (Mizelle \& Kritsky, 1969) - Cichla kelberi, gills.

Gussevia rogersi Kritsky, Thatcher \& Boeger, 1989 - Astronotus crassipinnis, gills. 
Gussevia undulata Kritsky, Thatcher \& Boeger, 1986 - Cichla kelberi, Cichla piquiti Kullander \& Ferreira, 2006, gills. Gussevia tucunarense Kritsky, Thatcher \& Boeger, 1986 - Cichla piquiti, gills.

Jainus sp. - Leporellus vittatus, Leporinus friderici, Leporinus lacustris, Megaleporinus piavussu, Moenkhausia aff. intermedia Eigenmann, 1908, Moenkhausia forestii, Salminus brasiliensis, Schizodon borellii, gills.

Jainus hexops Kritsky \& Leiby, 1972 - Moenkhausia forestii, Moenkhausia sanctaefilomenae (Steindachner, 1907), gills. Jainus piava Karling, Bellay, Takemoto \& Pavanelli, 2011 - Schizodon borellii, gills.

Kritskyia sp. - Megaleporinus obtusidens Valenciennes, 1836, Megaleporinus piavussu, swimming bladder and mesonephric duct.

Kritskyia annakohnae Boeger, Tanaka \& Pavanelli, 2001 - Serrasalmus maculatus, Serrasalmus marginatus, ureters and urinary bladder.

Kritskyia boegeri Lizama, Takemoto \& Pavanelli, 2002 - Prochilodus lineatus, urinary bladder.

Kritskyia eirasi Guidelli, Takemoto \& Pavanelli, 2003 - Leporinus friderici, Leporinus lacustris, urinary bladder and mesonephric duct.

Notothecium sp. - Serrasalmus marginatus, gills.

Notozothecium sp. - Serrasalmus marginatus, gills.

Omothecium sp. - Pinirampus pirinampu (Agassiz, 1829), gills.

Pavanelliella pavanellii Kritsky \& Boeger, 1998 - Pimelodus maculatus, nasal cavity and gills.

Rhinonastes pseudocapsaloideum Kritsky, Thatcher \& Boeger, 1988 - Prochilodus lineatus, nasal cavity.

Rhinoxenus sp. - Megaleporinus macrocephalus (Garavello \& Britski, 1988), Serrasalmus marginatus, gills.

Rhinoxenus arietinus Kritsky, Boeger \& Thatcher, 1988 - Leporinus friderici, Leporinus lacustris, Megaleporinus obtusidens, Megaleporinus piavussu, nasal cavity and gills.

Rhinoxenus bulbovaginatus Boeger, Domingues \& Pavanelli, 1995 - Salminus brasiliensis, nasal cavity.

Sciadicleithrum sp. - Crenicichla sp. gills.

Sciadicleithrum ergensi Kritsky, Thatcher \& Boeger, 1989 - Cichla piquiti, gills.

Sciadicleithrum joanae Yamada, Takemoto, Bellay \& Pavanelli, 2009 - Crenicichla britskii Kullander, 1982, gills.

Sciadicleithrum kritskyi Bellay, Takemoto, Yamada \& Pavanelli, 2009 - Geophagus sveni Lucinda, Lucena \& Assis, 2010, gills.

Sciadicleithrum paranaensis Bellay, Takemoto, Yamada \& Pavanelli, 2009 - Geophagus sveni, gills.

Sciadicleithrum uncinatum Kritsky, Thatcher \& Boeger, 1989 - Cichla piquiti, gills.

Sciadicleithrum satanopercae Yamada, Takemoto, Bellay \& Pavanelli, 2009 - Satanoperca sp., gills.

Tereancistrum sp. - Leporinus friderici, Leporinus lacustris, Megaleporinus obtusidens, Megaleporinus piavussu, Prochilodus lineatus, Schizodon borellii, gills.

Tereancistrum curimba Lizama, Takemoto \& Pavanelli, 2004 - Prochilodus lineatus, gills.

Tereancistrum paranaensis Karling, Lopes, Takemoto \& Pavanelli, 2014 - Schizodon borellii, gills.

Tereancistrum parvus Kritsky, Thatcher \& Kayton, 1980 - Leporinus friderici, Leporinus lacustris, Megaleporinus obtusidens, Megaleporinus piavussu, Schizodon borelli, gills.

Tereancistrum toksonum Lizama, Takemoto \& Pavanelli, 2004 - Prochilodus lineatus, gills.

Trinibaculum sp. - Schizodon borellii, gills.

Trinibaculum rotundus Karling, Lopes, Takemoto \& Pavanelli, 2011 - Schizodon borellii, gills.

Unibarra sp. - Pimelodus maculatus, Pinirampus pirinampu, Pseudoplatystoma corruscans, gills.

Urocleidoides sp. - Astyanax lacustris, Leporinus friderici, Leporinus lacustris, Megaleporinus obtusidens, Megaleporinus piavussu, Schizodon borellii, Steindachnerina insculpta (Fernández-Yépez, 1948), gills.

Urocleidoides brasiliensis Rosim, Mendoza-Franco \& Luque, 2011 - Hoplias spp., gills.

Urocleidoides cuiabai Rosim, Mendoza-Franco \& Luque, 2011 - Hoplias spp., gills.

Urocleidoides eremitus Kritsky, Thatcher \& Boeger, 1986 - Hoplias spp., gills.

Urocleidoides malabaricusi Rosim, Mendoza-Franco \& Luque, 2011 - Hoplias spp., gills. 
Urocleidoides paradoxus Kritsky, Thatcher \& Boeger, 1986 - Leporinus friderici, Leporinus lacustris, Megaleporinus obtusidens, Megaleporinus piavussu, gills.

Vancleaveus sp. - Pseudoplatystoma corruscans, Trachydoras paraguayensis, gills.

Vancleaveus fungulus Kritsky, Thatcher \& Boeger, 1986 - Pseudoplatystoma corruscans, gills.

Vancleaveus janauacaensis Kritsky, Thatcher \& Boeger, 1986 - Hoplias spp., gills.

\section{Family Gyrodactylidae Van Beneden \& Hesse, 1863}

Gyrodactylus sp. - Moenkhausia forestii, Prochilodus lineatus, gills and nasal cavity.

Gyrodactylus neotropicalis Kritsky \& Fritts, 1970 - Astyanax lacustris, Moenkhausia forestii, gills.

\section{DIGENEA}

\section{Phylum Platyhelminthes Minot, 1876 \\ Class Trematoda Rudolphi, 1808 \\ Subclass Digenea Carus, 1863}

Digenea fam. gen. sp. - Crenicichla britskii, Cyphocharax modestus (Fernández-Yépez, 1948), Gymnotus inaequilabiatus (Valenciennes, 1839), Hoplerythrinus unitaeniatus, Laetacara araguaiae, Loricariichthys rostratus Reis \& Pereira, 2000, Megaleporinus macrocephalus, Moenkhausia aff. intermedia, Moenkhausia forestii, Myloplus tiete (Eigenmann \& Norris, 1900), Pinirampus pirinampu, Pseudoplatystoma corruscans, Rhamdia quelen, Synbranchus marmoratus Bloch, 1795, adult and metacercariae, mesentery, eyes, cranial cavity, gills, muscle, swimming bladder and kidney.

\section{Order Diplostomida Olson, Cribb, Tkach, Bray \& Littlewood, 2003 Family Diplostomidae Poirier, 1886}

Austrodiplostomum sp. - Cichla piquiti, Hemisorubim platyrhynchos (Valenciennes, 1840), Plagioscion squamosissimus, Rhinelepis aspera, Sorubim lima (Bloch \& Schneider, 1801), metacercariae, eyes.

Austrodiplostomum compactum (Lutz, 1928) - Acestrorhynchus lacustris, Auchenipterus osteomystax, Cichla kelberi, Crenicichla britskii, Geophagus sveni, Hoplias spp., Hypostomus regani, Loricariichthys platymetopon, Pimelodus ornatus Kner, 1858, Plagioscion squamosissimus, Prochilodus lineatus, Pterygoplichthys ambrosettii (Holmberg, 1893), Satanoperca sp., Schizodon borellii, metacercariae, eyes.

Diplostomidae gen. sp. - Ageneiosus inermis (Linnaeus, 1766), Aphyocharax anisitsi, Auchenipterus osteomystax, Crenicichla sp., Cyphocharax nagelii (Steindachner, 1881), Geophagus brasiliensis, Hemisorubim platyrhynchos, Hypostomus ternetzi, Loricaria sp., Loricariichthys platymetopon, Loricariichthys rostratus, Megaleporinus piavussu, Psellogrammus kennedyi (Eigenmann, 1903), Pseudoplatystoma corruscans, Sorubim lima, Trachydoras paraguayensis, metacercariae, mesentery, eyes, cranial cavity, gills, muscle, swimming bladder and kidney.

Diplostomum sp. - Cichla kelberi, Cyphocharax nagelii, Gymnotus spp., Hoplerythrinus unitaeniatus, Hoplias spp., Leporinus friderici, Parauchenipterus galeatus, Pimelodus maculatus, Steindachnerina insculpta, metacercariae, eyes.

Neascus sp. - Crenicichla sp., metacercariae, encysted on the heart.

Neodiplostomum sp. - Gymnotus spp., Megaleporinus piavussu, metacercariae, visceral cavity.

Sphincterodiplostomum sp. - Cichla piquiti, Hemisorubim platyrhynchos, Prochilodus lineatus, Steindachnerina brevipinna (Eigenmann \& Eigenmann, 1889), metacercariae, eyes and visceral cavity.

Sphincterodiplostomum musculosum Dubois, 1936 - Hoplias spp., Steindachnerina insculpta, metacercariae, eyes and visceral cavity.

Tylodelphis sp. - Astyanax lacustris, Cyphocharax nagelii, Gymnotus spp., Leporinus lacustris, Potamotrygon cf. Falkneri Castex \& Maciel, 1963, Prochilodus lineatus, metacercariae, visceral cavity.

\section{Family Proterodiplostomidae Dubois, 1936}

Crocodilicola sp. - Auchenipterus osteomystax, Gymnotus spp., metacercariae, gonads.

Crocodilicola pseudostoma (Willemoes-Suhm, 1870) - Hemisorubim platyrhynchos, Loricariichthys platymetopon, adult, metacercariae and progenetic metacercariae, swimming bladder, visceral cavity and digestive tract.

Cystodiplostomum sp. - Leporinus lacustris, metacercariae, gonads. 
Herpetodiplostomum sp. - Astyanax lacustris, Leporinus friderici, Leporinus lacustris, Megaleporinus obtusidens, Megaleporinus piavussu, metacercariae, swimming bladder.

Herpetodiplostomum gymnoti Szidat \& Graefe, 1969 - Iheringichthys labrosus, metacercariae, mesentery and gonads.

\section{Family Sanguinicolidae von Graff, 1907}

Kritsky platyrhynchi (Guidelli, Isaac \& Pavanelli, 2002) - Hemisorubim platyrhynchos, adult, visceral cavity. Sanguinicola sp. - Megaleporinus piavussu, Sorubim lima, adult, visceral cavity.

Sanguinicola coelomica (Szidat, 1951) - Pimelodus maculatus, adult, coelom.

\section{Order Plagiorchiida La Rue, 1957}

Family Acanthostomidae Poche, 1926

Acanthostomum gnerii Szidat, 1954 - Rhamdia quelen, adult, intestine.

\section{Family Allocreadiidae Looss, 1902}

Auriculostoma platense (Szidat, 1954) - Iheringichthys labrosus, Pimelodus maculatus, adult, intestine.

Crepidostomum platense Szidat, 1954 - Pimelodus maculatus, adult, stomach.

Creptotrema sp. - Leporinus lacustris, adult, intestine.

Creptotrema creptotrema Travassos, Artigas \& Pereira, 1928 - Auchenipterus osteomystax, Leporellus vittatus, Leporinus friderici, Megaleporinus obtusidens, Pimelodus maculatus, adult, intestine.

Creptotrema lynchi Brooks, 1976 - Megaleporinus piavussu, adult, intestine.

Creptotrematina sp. - Moenkhausia forestii, adult, intestine.

\section{Family Apocreadiidae Skrjabin, 1942}

Crassicutis cichlasomae Manter, 1936 - Crenicichla sp., Geophagus brasiliensis, Loricaria sp., adult, intestine.

Crassicutis intermedius (Szidat, 1954) - Hypostomus regani, Hypostomus ternetzi, adult, intestine.

\section{Family Bucephalidae Poche, 1907}

Bucephalidae gen. sp. - Astyanax lacustris, eyes, brain, muscles and visceral cavity.

Prosorhynchus piranhus Thatcher, 1999 - Serrasalmus maculatus, adult, intestinal tract.

Rhipidocotyle gibsoni Kohn \& Fernandes, 1994 - Acestrorhynchus lacustris, adult, intestine.

Rhipidocotyle jeffersoni (Kohn, 1970) - Salminus brasiliensis, adult, intestine.

\section{Family Callodistomidae Odhner, 1910}

Prosthenhystera sp. - Hoplias spp., adult, gall bladder.

Prosthenhystera obesa (Diesing, 1850) - Pimelodus maculatus, Salminus brasiliensis, adult, gall bladder.

\section{Family Cladorchiidae Fischoeder, 1901}

Colocladorchis sp. - Prochilodus lineatus, adult, intestine.

Colocladorchis ventrastomis Thatcher, 1979 - Prochilodus lineatus, adult, intestine.

Dadaytrema sp. - Megalancistrus parananus, adult, intestine.

Dadaytrema oxycephala (Diesing, 1836) - Brycon orbignyanus (Valenciennes, 1850), Piaractus mesopotamicus, Pseudoplatystoma corruscans, Pterodoras granulosus (Valenciennes, 1821), adult, intestine.

Dadaytremoides parauchenipteri (Lunaschi, 1989) - Ageneiosus inermis, adult and metacercariae, stomach and intestine. Dadayius pacupeva Lacerda, Takemoto \& Pavanelli, 2003 - Metynnis lippincottianus, adult, intestinal tract.

\section{Family Cryptogonimidae Ciurea, 1933}

Iheringtrema iheringi Travassos, 1948 - Zungaro jahu (Ihering, 1898), adult, intestine.

Parspina sp. - Iheringichthys labrosus, adult, intestine.

Pseudosellacotyla lutzi (Freitas, 1941) - Hoplias spp., adult, intestine. 


\section{Family Derogenidae Nicoll, 1910}

Genarchella sp. - Potamotrygon cf. falkneri, adult, stomach.

Genarchella astyanactis (Watson, 1976) - Megaleporinus obtusidens, Plagioscion squamosissimus, adult, stomach.

Genarchella genarchella Travassos, Artigas \& Pereira, 1928 - Megalancistrus parananus, Pimelodus ornatus, Potamotrygon amandae, adult, buccal cavity, oesophagus and stomach.

Thometrema overstreeti (Brooks, Mayes \& Thorson, 1979) - Hoplias spp., Pimelodus maculatus, Salminus brasiliensis, adult, stomach.

\section{Family Didymozoidae Monticelli, 1888}

Didymozoidae gen. sp. - Moenkhausia forestii, eyes, brain, muscles and visceral cavity.

\section{Family Echinostomatidae Looss, 1899}

Echinostomatidae gen. sp. - Leporinus friderici, metacercariae, eyes, brain, muscles and visceral cavity.

\section{Family Faustulidae Poche, 1926}

Antorchis sp. - Astyanax lacustris, metacercariae, intestine.

\section{Family Gorgoderidae Looss, 1899}

Gorgoderidae gen. sp. - Hemisorubim platyrhynchos, adult, urinary bladder.

\section{Family Haploporidae Nicoll, 1914}

Chalcinotrema thatcheri Kohn, Fernandes \& Gibson, 1999 - Leporinus lacustris, Schizodon nasutus, adult, intestine. Lecithobothrioides sp. - Prochilodus lineatus, adult, intestine.

Megacoelium sp. - Megaleporinus piavussu, Prochilodus lineatus, adult, stomach.

Paralecithobothrys brasiliensis Freitas, 1948 - Leporinus friderici, Megaleporinus obtusidens, Megaleporinus piavussu, Schizodon borellii, Schizodon nasutus, adult, intestine.

Saccocoelioides sp. - Prochilodus lineatus, adult, pyloric caeca and intestine.

Saccocoelioides elongatus Szidat, 1954 - Prochilodus lineatus, adult, intestine.

Saccocoelioides godoyi Kohn \& Froes, 1986 - Cyphocharax nagelii, Leporinus friderici, adult, intestine and stomach. Saccocoelioides leporinodus Thatcher, 1978 - Prochilodus lineatus, adult, intestine.

Saccocoelioides magniovatus Szidat, 1954 - Megaleporinus obtusidens, adult, pyloric caeca and stomach.

Saccocoelioides magnorchis Thatcher, 1978 - Prochilodus lineatus, adult, pyloric caeca, intestine and stomach.

Saccocoelioides magnus Szidat, 1954 - Leporinus lacustris, Megalancistrus parananus, Megaleporinus obtusidens, Schizodon nasutus, adult, intestine.

Saccocoelioides nanii Szidat, 1954 - Prochilodus lineatus, Rhaphiodon vulpinus, adult, intestine.

Saccocoelioides platensis Lunaschi, 1984 - Schizodon borellii, adult, intestine.

Saccocoelioides saccodontis Thatcher, 1978 - Leporinus friderici, Prochilodus lineatus, adult, pyloric caeca and intestine. Unicoelium prochilodorum Thatcher \& Dossman, 1975 - Prochilodus lineatus, adult, intestine.

\section{Family Heterophyidae Odhner, 1914}

Ascocotyle sp. - Astyanax lacustris, Cichla piquiti, Geophagus sveni, Moenkhausia forestii, Satanoperca sp., Serrasalmus marginatus, metacercariae, body musculature, heart, stomach, liver, kidney, spleen, gonads and mesentery.

\section{Family Macroderoididae McMullen, 1937}

Magnivitellinum corvitellinum Lacerda, Takemoto \& Pavanelli, 2009 - Hoplosternum littorale (Hancock, 1828), adult, digestive tract.

Magnivitellinum simplex Kloss, 1966 - Roeboides descalvadensis, adult, intestine.

\section{Family Microscaphidiidae Looss, 1900}

Curumai curumai Travassos, 1961 - Piaractus mesopotamicus, adult, intestine. 


\section{Family Opisthorchiidae Braun, 1901}

Neocladocystis intestinalis (Vaz, 1932) - Salminus brasiliensis, adult, intestine.

Family Paramphistomidae Fischoeder, 1901

Microrchis oligovitellum Lunaschi, 1987 - Auchenipterus osteomystax, Parauchenipterus galeatus, adult, intestine.

\section{CESTODA}

Phylum Platyhelminthes Minot, 1876

Class Cestoda Rudolphi, 1808

Cestoda fam. gen. sp. - Acestrorhynchus lacustris, Crenicichla jaguarensis Haseman, 1911, Galeocharax gulo (Cope, 1870), Gymnotus inaequilabiatus, Potamotrygon amandae, Piaractus mesopotamicus, Plagioscion squamosissimus, Sternopygus macrurus (Bloch \& Schneider, 1801), Salminus brasiliensis, Schizodon borellii, plerocercoid and adult, digestive tract.

\section{Order Bothriocephalidea Kuchta, Scholz, Brabec \& Bray, 2008}

\section{Family Bothriocephalidae Blanchard, 1849}

Regobothrium microhamulinum Scholz, Takemoto \& Kuchta, 2017 - Catathyridium jenynsii (Gunther, 1862), Ageneiosus ucayalensis Castelnau, 1855, adult, intestine.

Senga sp. - Ageneiosus inermis, adult, intestine.

\section{Order Cyclophyllidea Van Beneden in Braun, 1900}

Family Gryporhynchidae Spassky \& Spasskaya, 1973

Valipora sp. - Crenicichla britskii, Pimelodus maculatus, plerocercoid, gall bladder.

Valipora campylancristrota (Wedl, 1855) - Prochilodus lineatus, plerocercoid and adult, gall bladder.

\section{Order Onchoproteocephalidea Caira, Jensen, Waeschenbach, Olson \& Littlewood, 2014}

Onchoproteocephalidea gen. sp. - Acestrorhynchus lacustris, Aphyocharax anisitsi, Astyanax lacustris, Galeocharax gulo, Iheringichthys labrosus, Leporellus vittatus, Prochilodus lineatus, Psellogrammus kennedyi, Satanoperca sp., Serrasalmus maculatus, Steindachnerina insculpta, plerocercoid and adult, digestive tract.

\section{Family Onchobothriidae Braun, 1900}

Acanthobothrium regoi Brooks, Mayes \& Thorson, 1981 - Potamotrygon amandae, Potamotrygon cf. falkneri, adult, spiral valve.

Ageneiella brevifilis Chambrier \& Vaucher, 1999 - Ageneiosus inermis, adult, intestine.

Cangatiella arandasi Pavanelli \& Santos, 1991 - Parauchenipterus galeatus, adult, intestine.

Chambriella agostinhoi (Pavanelli \& Santos, 1992) - Zungaro jahu, adult, intestine.

Chambriella itaipuensis (Pavanelli \& Rego, 1991) - Hemisorubim platyrhynchos, Sorubim lima, adult, intestine.

Chambriella paranaensis (Pavanelli \& Rego, 1989) - Hemisorubim platyrhynchos, adult, intestine.

Choanoscolex abscissus (Riggenbach, 1895) - Pseudoplatystoma corruscans, Zungaro jahu, adult, intestine.

Gibsoniela sp. - Ageneiosus inermis, adult, intestine.

Goezeella nupeliensis Pavanelli \& Rego, 1991 - Sorubim lima, adult, intestine.

Harriscolex kaparari (Woodland, 1935) - Pseudoplatystoma corruscans, adult, intestine.

Jauella glandicephala Rego \& Pavanelli, 1985 - Zungaro jahu, adult, intestine.

Manaosia bracodemoca Woodland, 1935 - Sorubim lima, adult, intestine.

Mariauxiella pimelodi Chambrier \& Rego, 1995 - Pimelodus ornatus, Sorubim lima, adult, intestine.

Mariauxiella piscatorum Chambrier \& Vaucher, 1999 - Hemisorubim platyrhynchos, adult, intestine.

Megathylacus brooksi Rego \& Pavanelli, 1985 - Zungaro jahu, adult, intestine.

Megathylacus travassosi Pavanelli \& Santos, 1991 - Pseudoplatystoma corruscans, adult, intestine.

Monticellia belavistensis Pavanelli, Machado, Takemoto \& Santos, 1994 - Piaractus mesopotamicus, adult, intestine. 
Monticellia coryphicephala (Monticelli, 1892) - Salminus brasiliensis, adult, intestine.

Monticellia loyolai Pavanelli \& Santos, 1992 - Pimelodus maculatus, adult, intestine.

Monticellia magna (Rego, dos Santos \& Silva, 1974) - Pimelodus maculatus, adult, intestine.

Nomimoscolex sp. - Pimelodus maculatus, adult, intestine.

Nomimoscolex admonticellia (Woodland, 1934) - Pinirampus pirinampu, adult, intestine.

Nomimoscolex chubbi (Pavanelli \& Takemoto, 1995) - Gymnotus spp., Gymnotus inaequilabiatus, adult, intestine.

Nomimoscolex pertierrae Chambrier, Takemoto \& Pavanelli, 2006 - Pseudoplatystoma corruscans, adult, intestine.

Nomimoscolex sudobim Woodland, 1935 - Pseudoplatystoma corruscans, adult, intestine.

Nupelia portoricensis Pavanelli \& Rego, 1991 - Hemisorubim platyrhynchos, Sorubim lima, adult, intestine.

Peltidocotyle rugosa Diesing, 1850 - Zungaro jahu, adult, intestine.

Potamotrygonocestus travassosi Rego, 1979 - Potamotrygon cf. falkneri, adult, spiral valve.

Proteocephalinae gen. sp. - Aphyocharax anisitsi, Galeocharax gulo, Iheringichthys labrosus, Moenkhausia forestii, Prochilodus lineatus, Satanoperca sp., Serrasalmus maculatus, Steindachnerina insculpta, plerocercoid and adult, digestive tract.

Proteocephalus macrophallus (Diesing, 1850) - Cichla kelberi, Cichla piquiti, adult, intestine.

Proteocephalus microscopicus (Woodland, 1935) - Cichla kelberi, Cichla piquiti, adult, intestine.

Proteocephalus serrasalmus Rego \& Pavanelli, 1990 - Serrasalmus maculatus, adult, intestine.

Proteocephalus vazzolerae Pavanelli \& Takemoto, 1995 - Leporinus friderici, Leporinus lacustris, Piaractus mesopotamicus, adult, intestine.

Rudolphiella piranabu (Woodland, 1934) - Pinirampus pirinampu, adult, intestine.

Sciadocephalus megalodiscus Diesing, 1850 - Cichla kelberi, Cichla piquiti, adult, intestine.

Spasskyellina sp. - Pimelodus ornatus, plerocercoid and adult, intestine.

Spasskyellina mandi Pavanelli \& Takemoto, 1996 - Pimelodus ornatus, adult, intestine.

Spasskyellina spinulifera (Woodland, 1935) - Ageneiosus inermis, Brycon orbignyanus, Pseudoplatystoma corruscans, Sorubim lima, plerocercoid and adult, intestine.

Spatulifer maringaensis Pavanelli \& Rego, 1989 - Hemisorubim platyrhynchos, Sorubim lima, adult, intestine.

Travassiella avitellina Rego \& Pavanelli, 1987 - Zungaro jahu, adult, intestine.

Order Rhinebothriidea Healy, Caira, Jensen, Webster \& Littlewood, 2009

Family Eutetrarhynchidae Guiart, 1927

Paroncomegas araya (Woodland, 1934) - Potamotrygon cf. falkneri, adult, spiral valve.

\section{Family Rhinebothriidae Euzet, 1953}

Rhinebothrium paratrygoni Rego \& Dias, 1976 - Potamotrygon amandae, Potamotrygon cf. falkneri, adult, spiral valve.

\section{NEMATODA}

\section{Phylum Nematoda Rudolphi, 1808}

Nematoda fam. gen. sp. - Aphyocharax anisitsi, Crenicichla britskii, Hypophthalmus oremaculatus, Hypostomus regani, Moenkhausia forestii, Pinirampus pirinampu, Prochilodus lineatus, Rhamphichthys hahni (Meinken, 1937), Rhinodoras dorbignyi (Kner, 1855), Satanoperca sp., immature form and adult, digestive tract.

\section{Class Adenophorea Linstow, 1905}

Order Enoplida Schuurmans, Stekhoven \& Deconinck, 1933

Family Dioctophymatidae (Railliet, 1915)

Eustrongylides sp. - Acestrorhynchus lacustris, Cichla kelberi, Gymnotus spp., Lepthoplosternum pectorale (Boulenger, 1895), Megaleporinus piavussu, Pimelodus maculatus, Serrasalmus maculatus, Sorubim lima, immature form, mesentery, abdominal cavity, musculature, liver and gonads. 
Eustrongylides ignotus Jägerskiöld, 1909 - Ageneiosus inermis, Hemisorubim platyrhynchos, Hoplias spp., Pseudoplatystoma corruscans, Serrasalmus maculatus, Salminus brasiliensis, Serrasalmus marginatus, immature form and adult, mesentery, abdominal cavity, musculature, gonads and liver.

\section{Family Trichuridae Railliet, 1915}

Capillariidae gen. sp. - Pseudoplatystoma corruscans, Serrasalmus maculatus, Serrasalmus marginatus, adult, intestine. Capillostrongyloides sentinosa (Travassos, 1927) - Megaleporinus obtusidens, adult, pyloric caeca.

Paracapillaria piscicola (Travassos, Artigas \& Pereira, 1928) - Hoplias spp., Salminus brasiliensis, adult, stomach and intestine.

\section{Class Secernentea (von Linstow, 1905) \\ Order Ascaridida Yamaguti, 1961 \\ Family Anisakidae Skrjabin \& Karokhin, 1945}

Anisakidae gen. sp. - Brycon hilarii Valenciennes, 1850, Loricaria sp., Megaleporinus obtusidens, Prochilodus lineatus, Salminus brasiliensis, immature form, intestine.

Contracaecum sp. - Acestrorhynchus lacustris, Ageneiosus inermis, Astyanax lacustris, Brycon orbignyanus, Cichla kelberi, Cichla piquiti, Crenicichla sp., Galeocharax gulo, Gymnotus spp., Hemisorubim platyrhynchos, Hoplias spp., Iheringichthys labrosus, Leporinus friderici, Leporinus lacustris, Leporellus vittatus, Megaleporinus obtusidens, Megaleporinus piavussu, Metynnis lippincottianus, Moenkhausia forestii, Parauchenipterus galeatus, Pimelodus maculatus, Plagioscion squamosissimus, Prochilodus lineatus, Psellogrammus kennedyi, Pseudoplatystoma corruscans, Rhaphiodon vulpinus, Salminus brasiliensis, Serrasalmus maculatus, Serrasalmus marginatus, Sorubim lima, immature form, abdominal cavity, mesentery and liver.

Goezia sp. - Ageneiosus militaris Valenciennes, 1836, Brycon orbignyanus, Hemisorubim platyrhynchos, Iheringichthys labrosus, Parauchenipterus galeatus, Piaractus mesopotamicus, Pimelodus maculatus, Rhaphiodon vulpinus, Serrasalmus marginatus, Sorubim lima, immature form and adult, stomach and intestine.

Goezia brasiliensis Moravec, Kohn \& Fernandes, 1994 - Brycon hilarii, Pseudoplatystoma corruscans, adult, stomach and intestine.

Goezia brevicaeca Moravec, Kohn \& Fernandes, 1994 - Brycon hilarii, Leporinus friderici, adult, stomach.

Goezia spinulosa (Diesing, 1839) - Hoplias spp., Leporinus friderici, Megaleporinus obtusidens, Megaleporinus piavussu, adult, stomach and pyloric caeca.

Hysterothylacium sp. - Brycon orbignyanus, Crenicichla sp., Galeocharax gulo, Geophagus brasiliensis, Gymnotus spp., Hypophthalmus oremaculatus, Leporinus friderici, Piaractus mesopotamicus, Plagioscion squamosissimus, Rhaphiodon vulpinus, Salminus brasiliensis, Serrasalmus marginatus, immature form and adult, intestine.

Raphidascaris sp. - Prochilodus lineatus, adult, intestine and stomach.

Raphidascaris (Sprentascaris) sp. - Geophagus brasiliensis, Geophagus sveni, immature form, intestine.

Raphidascaris (Sprentascaris) hypostomi (Petter \& Cassone, 1984) - Hypostomus ternetzi, adult, intestine.

Raphidascaris (Sprentascaris) lanfrediae Melo, Santos, Giese, Santos \& Santos, 2011 - Ageneiosus inermis, adult, intestine.

Raphidascaris (Sprentascaris) mahnerti (Petter \& Cassone, 1984) - Loricaria sp., Loricariichthys platymetopon, Loricariichthys rostratus, Metynnis lippincottianus, adult, digestive tract.

\section{Family Ascarididae Baird, 1853}

Brevimulticaecum sp. - Leporinus friderici, Leporinus lacustris, Megaleporinus obtusidens, Megaleporinus piavussu, Moenkhausia forestii, Potamotrygon cf. falkneri, immature form, abdominal cavity, intestine, stomach and liver.

Porrocaecum sp. - Hoplias spp., Megaleporinus obtusidens, immature form, swimming bladder.

\section{Family Atractidae Travassos, 1919}

Klossinemella sp. - Pimelodus maculatus, adult, intestine.

Rondonia rondoni Travassos, 1920 - Piaractus mesopotamicus, Pterodoras granulosus, Rhaphiodon vulpinus, Trachydoras paraguayensis, adult, intestine. 


\section{Family Cucullanidae Cobbold, 1864}

Cucullanus sp. - Potamotrygon cf. falkneri, Rhaphiodon vulpinus, Serrasalmus maculatus, Serrasalmus marginatus, Zungaro jahu, adult, intestine.

Cucullanus (Cucullanus) brevispiculus Moravec, Kohn \& Fernandes, 1993 - Ageneiosus inermis, Auchenipterus osteomystax, adult, intestine.

Cucullanus (Cucullanus) pinnai pinnai Travassos, Artigas \& Pereira, 1928 - Ageneiosus militaris, Hemisorubim platyrhynchos, Loricaria sp., Pimelodus maculatus, Pimelodus ornatus, Schizodon borellii, Sorubim lima, Zungaro jahu, adult, intestine.

Cucullanus (Cucullanus) pinnai pterodorasi Moravec, Kohn \& Fernandes, 1997 - Piaractus mesopotamicus, adult, intestine.

Cucullanus (Cucullanus) pseudoplatystomae Moravec, Kohn \& Fernandes, 1993 - Pseudoplatystoma corruscans, adult, intestine.

Cucullanus (Cucullanus) zungaro Vaz \& Pereira, 1934 - Hemisorubim platyrhynchos, Zungaro jahu, adult, intestine.

Dichelyne (Cucullanellus) sp. - Parauchenipterus galeatus, immature form, intestine.

Dichelyne leporini Petter, 1989 - Leporinus friderici, Leporinus lacustris, adult, intestine.

Dichelyne pimelodi Moravec, Kohn \& Fernandes, 1997 - Pimelodus maculatus, adult, intestine.

Seuratoidea gen. sp. - Zungaro jahu, adult, digestive tract.

\section{Family Kathlaniidae Lane, 1914}

Spectatus sp. - Piaractus mesopotamicus, immature form, intestine.

\section{Family Quimperiidae Gendre, 1928}

Neoparaseuratum travassosi Moravec, Kohn \& Fernandes, 1992 - Piaractus mesopotamicus, Trachydoras paraguayensis, adult, intestine.

\section{Order Oxyurida Chabaud, 1974}

\section{Family Pharyngodonidae Travassos, 1919}

Cosmoxynema vianai Travassos, 1949 - Steindachnerina insculpta, adult, intestine.

Cosmoxynemoides sp. - Cyphocharax nagelii, Trachydoras paraguayensis, adult, intestine.

Ichthyouris sp. - Brycon orbignyanus, Sorubim lima, adult, intestine.

Ichthyouris brasiliensis Moravec, Kohn \& Fernandes, 1992 - Megalancistrus parananus, Plagioscion squamosissimus, adult, intestine.

Ichthyouris laterifilamenta Moravec, Kohn \& Fernandes, 1992 - Trachydoras paraguayensis, adult, intestine.

Oxyuroidea gen. sp, Pinirampus pirinampu, adult, intestine.

Parasynodontisia petterae Moravec, Kohn \& Fernandes, 1992 - Rhinelepis aspera, Trachydoras paraguayensis, adult, intestine.

Spinoxyuris oxydoras Petter, 1994 - Ageneiosus inermis, Metynnis lippincottianus, adult, intestine.

Travnema travnema Pereira, 1938 - Steindachnerina insculpta, adult, intestine.

\section{Order Spirurida Chitwood, 1933}

Family Acuariidae Railliet, Henry \& Sisoff, 1912

Acuariidae gen. sp. - Salminus brasiliensis, immature form, mesentery.

\section{Family Camallanidae Railliet \& Henry, 1915}

Paracamallanus amazonensis Ferraz \& Thatcher, 1992 - Hypophthalmus oremaculatus, Piaractus mesopotamicus, Plagioscion squamosissimus, adult, intestine.

Procamallanus sp. - Acestrorhynchus lacustris, Schizodon altoparanae (Garavello \& Britski, 1990), Trachydoras paraguayensis, adult, intestine. 
Procamallanus (Procamallanus) annipetterae Kohn \& Fernandes, 1988 - Hypostomus regani, Hypostomus ternetzi, Megalancistrus parananus, adult, intestine

Procamallanus (Procamallanus) peraccuratus Pinto, Fábio, Noronha \& Rolas, 1976 - Crenicichla sp., Geophagus brasiliensis, Hemisorubim platyrhynchos, Hoplias spp., Potamotrygon amandae, adult, intestine and occasionally stomach.

Procamallanus (Spirocamallanus) sp. - Auchenipterus osteomystax, Geophagus brasiliensis, Pimelodus maculatus, Pseudoplatystoma corruscans, adult, intestine.

Procamallanus (Spirocamallanus) amarali Vas \& Pereira, 1934 - Leporinus friderici, Megaleporinus obtusidens, Megaleporinus piavussu, adult, intestine and pyloric caeca.

Procamallanus (Spirocamallanus) caballeroi (Bashirullah, 1977) - Astyanax lacustris, adult, intestine.

Procamallanus (Spirocamallanus) hilarii Vaz \& Pereira, 1934 - Brycon orbignyanus, Hemisorubim platyrhynchos, adult, intestine.

Procamallanus (Spirocamallanus) iheringi Travassos, Artigas \& Pereira, 1928 - Leporinus friderici, Megaleporinus obtusidens, Megaleporinus piavussu, Schizodon borellii, adult, intestine and pyloric caeca.

Procamallanus (Spirocamallanus) inopinatus Travassos, Artigas \& Pereira, 1928 - Ageneiosus inermis, Brycon orbignyanus, Crenicichla jaguarensis, Hoplias spp., Leporinus friderici, Megaleporinus obtusidens, Megaleporinus piavussu, Metynnis lippincottianus, Parauchenipterus galeatus, Potamotrygon amandae, Piaractus mesopotamicus, Schizodon borellii, Serrasalmus marginatus, Sorubim lima, Trachydoras paraguayensis, adult, intestine and occasionally stomach.

Procamallanus (Spirocamallanus) neocaballeroi (Caballero-Deloya, 1977) - Serrasalmus maculatus, Serrasalmus marginatus, adult, intestine.

Procamallanus (Spirocamallanus) paraguayensis (Petter, 1990) - Brycon orbignyanus, adult, intestine.

Procamallanus (Spirocamallanus) pimelodus Pinto, Fábio, Noronha \& Rolas, 1975 - Iheringichthys labrosus, Pimelodus maculatus, adult, intestine.

Procamallanus (Spirocamallanus) rarus Travassos, Artigas \& Pereira, 1928 - Cichla piquiti, Sorubim lima, adult, intestine.

\section{Family Cystidicolidae Skrjabin, 1946}

Cystidicoloides sp. - Leporinus friderici, immature form, mesentery.

Spinitectus sp. - Potamotrygon cf. falkneri, adult, spiral valve.

Spinitectus asperus Travassos, Artigas \& Pereira, 1928 - Prochilodus lineatus, adult, intestine.

\section{Family Gnathostomatidae (Railliet, 1895)}

Ancyracanthus schubarti (Kohn, Gomes \& Motta, 1968) - Leporinus lacustris, Schizodon borellii, adult, stomach and intestine.

Echinocephalus sp. - Potamotrygon cf. falkneri, adult, spiral valve.

Spiroxys sp. - Astyanax lacustris, Gymnotus spp., Moenkhausia forestii, Serrasalmus marginatus, immature form, abdominal cavity, mesentery and intestine.

\section{Family Guyanemidae Petter, 1974}

Guyanema raphiodoni Moravec, Kohn \& Fernandes, 1993 - Rhaphiodon vulpinus, adult, intestine.

Travassosnema travassosi paranaensis Moravec, Kohn \& Fernandes, 1993 - Acestrorhynchus lacustris, adult, abdominal cavity.

\section{Family Philometridae Baylis \& Daubney, 1926}

Philometra sp. - Pimelodus maculatus, adult, gonads and intestine.

Philometridae gen. sp. - Acestrorhynchus lacustris, Pseudoplatystoma corruscans, Serrasalmus maculatus, Serrasalmus marginatus, adult, abdominal cavity.

\section{Family Rhabdochonidae Skrjabin, 1946}

Johnstonmawsonia sp. - Auchenipterus osteomystax, Parauchenipterus galeatus, adult, intestine.

Rhabdochona acuminata Molin, 1860 - Auchenipterus osteomystax, Brycon orbignyanus, adult, intestine. 


\section{ACANTHOCEPHALA}

Phylum Acanthocephala Kölreuter, 1771

Acanthocephala fam. gen. sp. - Ageneiosus inermis, Ageneiosus ucayalensis, Brycon orbignyanus, Cichlasoma paranaense Kullander, 1983, Hypostomus cochliodon Kner, 1854, Hypostomus regani, Loricaria sp., Piaractus mesopotamicus, Pseudoplatystoma corruscans, Schizodon altoparanae, Schizodon nasutus, Serrasalmus maculatus, Serrasalmus marginatus, digestive tract.

Class Palaeacanthocephala Meyer, 1931

Order Echinorhynchida Southwell \& MacFie, 1925

Family Echinorhynchidae Cobbold, 1876

Echinorhynchus sp. - Schizodon borellii, Serrasalmus marginatus, adult, intestine.

Echinorhynchus briconi Machado-Filho, 1959 - Brycon orbignyanus, adult, intestine.

Echinorhynchus salobrensis Machado-Filho, 1948 - Serrasalmus maculatus, adult, intestine.

\section{Class Eoacanthocephala Van Cleve, 1936}

Order Gyracanthocephala Van Cleave, 1936

Family Quadrigyridae Van Cleave, 1920

Quadrigyrus sp. - Aphyocharax anisitsi, Astyanax lacustris, Iheringichthys labrosus, Moenkhausia forestii, Prochilodus lineatus, Psellogrammus kennedyi, Rhaphiodon vulpinus, immature form and adult, intestine.

Quadrigyrus brasiliensis Machado Filho, 1941 - Hoplerythrinus unitaeniatus, Steindachnerina insculpta, adult, intestine.

Quadrigyrus machadoi Fábio, 1983 - Hoplias spp., Cichla kelberi, Gymnotus spp., Hemisorubim platyrhynchos, Pimelodus maculatus, Potamotrygon amandae, Potamotrygon cf. falkneri, Sorubim lima, immature form and adult, intestine.

Quadrigyrus torquatus Van Cleave, 1920 - Acestrorhynchus lacustris, Leporinus lacustris, adult, intestine.

\section{Order Neoechinorhynchida Southwell \& MacFie, 1925}

\section{Family Neoechinorhynchidae Ward, 1917}

Gorytocephalus sp. - Hypostomus ternetzi, adult, intestine.

Neoechinorhynchus sp. - Pimelodus maculatus, Plagioscion squamosissimus, adult, intestine.

Neoechinorhynchus curemai Noronha, 1973 - Prochilodus lineatus, adult, intestine and pyloric caeca.

Neoechinorhynchus prochilodorum Nickol \& Thatcher, 1971 - Prochilodus lineatus, adult, intestine.

Octospiniferoides sp. - Brycon orbignyanus, adult, intestine.

Octospiniferoides incognita Schmidt \& Hugghins, 1973 - Leporinus lacustris, Schizodon borellii, Salminus brasiliensis, Brycon orbignyanus, adult, intestine.

\section{HIRUDINEA}

Phylum Annelida Lamarck, 1809

Class Clitellata Michaelsen, 1919

Order Rhynchobdellida Blanchard, 1894

Rhynchobdellida gen. sp. - Prochilodus lineatus, gills, mouth and fins.

\section{Family Glossiphoniidae Vaillant, 1890}

Helobdella sp. - Pimelodus maculatus, adult, gills, mouth and fins.

Family Piscicolidae Johnston, 1865

Myzobdella sp. - Pimelodus maculatus, adult, gills, mouth and fins.

\section{CRUSTACEA}

\section{Branchiura}

Phylum Arthropoda Siebold, 1848

Subphylum Crustacea Brünnich, 1772

Class Ichthyostraca Zrzavý, Hypša \& Vlášková, 1997 


\section{Subclass Branchiura Thorell, 1864}

Order Arguloida Yamaguti, 1963

Argulus sp. - Leporinus friderici, Leporinus lacustris, Megaleporinus piavussu, adult, body surface, nasal cavity and gills. Argulus pestifer Ringuelet, 1948 - Pseudoplatystoma corruscans, adult, body surface.

Dolops sp. - Megaleporinus obtusidens, Megaleporinus piavussu, Salminus brasiliensis, Schizodon borellii, adult, body surface, nasal cavity and gills.

Dolops carvalhoi Lemos de Castro, 1949 - Pseudoplatystoma corruscans, adult, body surface.

Dolops geayi Bouvier, 1897 - Prochilodus lineatus, adult, body surface.

Dolops longicauda Heller, 1857 - Salminus brasiliensis, adult, body surface and gills.

Dolops nana Lemos de Castro, 1950 - Leporinus friderici, Megaleporinus piavussu, Megaleporinus obtusidens, adult, body surface and gills.

\section{Copepoda}

Phylum Arthropoda Siebold, 1848

Subphylum Crustacea Brünnich, 1772

Class Copepoda Milne-Edwards, 1840

Copepoda fam. gen. sp. - Auchenipterus osteomystax, Eigenmannia trilineata López \& Castello, 1966, Gymnotus sylvius Albert \& Fernandes-Matioli, 1999, Moenkhausia forestii, Moenkhausia aff. intermedia, Satanoperca sp., nasal cavity and gills.

\section{Order Cyclopoida Burmeister, 1834}

\section{Family Ergasilidae von Nordmann, 1832}

Ergasilidae gen. sp. - Astyanax lacustris, Auchenipterus osteomystax, Leporinus friderici, Leporinus lacustris, Megaleporinus obtusidens, Megaleporinus piavussu, Pimelodus maculatus, Pinirampus pirinampu, Prochilodus lineatus, Pseudoplatystoma corruscans, adult, nasal cavity and gills.

Acusicola sp. - Astyanax lacustris, Prochilodus lineatus, adult, gills.

Amplexibranchius sp. - Megaleporinus piavussu, Prochilodus lineatus, adult, gills.

Brasergasilus sp. - Astyanax lacustris, adult, gills.

Ergasilus bryconis Thatcher, 1981 - Leporinus lacustris, Megaleporinus obtusidens, Megaleporinus piavussu, adult, gills. Ergasilus jaraquensis Thatcher \& Robertson, 1982 - Prochilodus lineatus, adult, gills.

Gamidactylus sp. - Pimelodus maculatus, Prochilodus lineatus, adult, nasal cavity.

Gamidactylus jaraquensis Thatcher \& Boeger, 1984 - Prochilodus lineatus, adult, nasal cavity.

Gamispatulus schizodontis Thatcher \& Boeger, 1984 - Leporinus friderici, Leporinus lacustris, Megaleporinus obtusidens, Megaleporinus piavussu, Piaractus mesopotamicus, Schizodon borellii, Serrasalmus maculatus, Serrasalmus marginatus, adult, nasal cavity.

Gamispinus diabolicus Thatcher \& Boeger, 1984 - Piaractus mesopotamicus, adult, nasal cavity.

Miracetyma sp. - Prochilodus lineatus, adult, gills.

Rhinergasilus sp. - Prochilodus lineatus, adult, nasal cavity.

Rhinergasilus piranhus Boeger \& Thatcher, 1998 - Acestrorhynchus lacustris, Serrasalmus maculatus, adult, nasal cavity. Therodamas sp. - Pimelodus maculatus, adult, gills.

Vaigamus sp. - Astyanax lacustris, Pimelodus maculatus, adult, gills and nasal cavity.

\section{Isopoda}

Phylum Arthropoda Siebold, 1848

Subphylum Crustacea Brünnich, 1772

Class Malacostraca Latreille, 1802

Order Isopoda Latreille, 1817

Cymothooidea fam. gen sp. - Cichla kelberi, Galeocharax gulo, Hoplias spp., adult, gills. 


\title{
Family Cymothoidae Leach, 1818
}

Telotha sp. - Pimelodus maculatus, adult, gills.

\section{Pentastomida}

Phylum Arthropoda Siebold, 1848

Subphylum Crustacea Brünnich, 1772

Class Ichthyostraca Zrzavý, Hypša \& Vlášková, 1997

Subclass Pentastomida Diesing, 1836

Order Porocephalida Heymons, 1935

Family Sebekiidae Fain, 1961

Sebekia oxycephala Diesing, 1835 - Hoplias spp., larvae, body surface.

\section{Family Subtriquetridae Fain, 1961}

Subtriquetra subtriquetra (Diesing, 1836) - Sorubim lima, larvae, swimming bladder.

\author{
ACARI \\ Phylum Arthropoda Siebold, 1848 \\ Class Arachnida Cuvier, 1812 \\ Order Acari Leach, 1817
}

Acarina fam. gen. sp. - Geophagus sveni, Serrasalmus marginatus, adult, gills and body surface.

\section{Discussion}

Our results indicate an increase of $68 \%$ in the number of identified species in the study area and a reduction of $6 \%$ of the taxonomic groups when compared with Takemoto et al. (2009). This result may be linked to an increase in the number of studies using integrative taxonomy for the identification of parasite species in this environment (e.g. Gasques et al., 2016; Da Graça et al., 2018; Franceschini et al., 2018).

The Monogenea class presented the largest number of identified species, and this, possibly, occurred due to the number of studies that have been carried out with the group in the floodplain. The group's richness has already been addressed by Takemoto et al. (2009), and our result can be explained by the identifications and descriptions carried out with the aid of technology, for example, use of molecular techniques, consequently, generating greater interest in the group, more studies and publications (Eiras et al., 2011).

Parasites belonging to the phylum Nematoda and to the subclass Digenea were the groups with the largest number of cataloged interactions, which may be associated with the fact that they exploit different hosts species in their life cycles (Esch et al., 2002; Poulin, 2002; Luque, 2004; Takemoto et al., 2009). In the Cestoda class, it was possible to observe a significant increase in the number of identified species and interactions with several hosts, which is in accordance with studies that classify the order Onchoproteocephalidae, for example. That study covers the largest number of parasites in freshwater fish species (Luque, 2004); however, studies on ecological and pathological aspects of their cycle are lacking (Pavanelli et al., 2013).

The species of the phylum Acanthocephala were found in low richness in this study. It is important to study these organisms because they are commonly used as bioindicators, due to their high capacity for absorbing trace elements (Taraschewski, 2000; Dos Reis et al., 2017). They have a large bioaccumulation capacity, especially due to the absence of a digestive system, which leads them to absorb the pre-digested nutrients through diffusion directly from the intestinal content of fish. In addition, the location of the parasite in the fish - as well as its developmental stage - can perform an important role in the bioaccumulation process (Nachev \& Sures, 2016; Paller et al., 2016; Sures et al., 2017).

The Hirudinea subclass has few records of interactions and it is still debated whether they are ectoparasites or associated organisms (Richardson et al., 2015). However, they play an important role in acting as vectors of flagellated protozoa of the Trypanosomatidae family, which were recorded parasitizing different hosts in our study. Much has been discussed about the specificity of Trypanosoma spp. and future studies with descriptions of new species for new hosts, using molecular biology, can help to understand these important parasites (Marchiori \& Martins, 2013).

The Myxozoa parasites recorded just three species parasitizing three different hosts, all native to the Paraná River. However, we can highlight here the importance of studying their hosts (vertebrates and invertebrates) more thoroughly, in association with these parasites, given that more recent studies have showed their life cycles with several hosts (Takemoto et al., 2009; Eiras \& Adriano, 2013). 
The phylum Arthropoda represents the greatest diversity of parasite groups (Arguloida, Pentastomida, Copepoda, Isopoda and Acarina), with a wide diversity of sizes, shapes and strategies of parasitism. In most of the cases, they are ectoparasites and are found in the gills and external surface of fish (Arguloida, Isopoda and Acarina), and also in some cavities of the body, such as nostrils (Copepoda), while the parasites of the subclass Pentastomida can be found in internal organs such as the gas bladder (Eiras et al., 2010). Although there is a large number of studies with these different groups, there is a lack of ecological studies (Luque et al., 2013), and the data published in this work partly reflect the lack of interest in studies on these groups acting as parasites.

In relation to infected host species, there was an increase of $14 \%$ in studies on their parasitic fauna in relation to the previous work, which can be explained by the constant process of invasion of non-native species of fish. The floodplain is located between two large reservoirs that, despite favoring the development of the local and regional economy, have seriously affected the aquatic biota with the emergence of non-native species (Gois et al., 2015; Agostinho et al., 2016). Invasive fish may be hosted by parasites that are not present in the local biota, and when introduced to the site together with their hosts, they can increase competition and affect the parasitic composition of native fish. In addition, it is known that $45 \%$ of fish species (Ota et al., 2018) whose parasitic fauna have already been studied are not native to the floodplain.

The biological characteristics of parasites have revealed information about host ecology, biogeography and phylogeny (Gardner \& Campbell, 1992; Raga et al., 2008), and have also provided indicators of current and historical ecological relationships (Aznar et al., 2001). Therefore, to understand the role of the parasite community in an ecosystem, it is necessary to have prior knowledge of the species that belong to it (Takemoto et al., 2009). Although the studies conducted in the upper Paraná River floodplain have contributed towards surveying the parasite species, the species richness is still underestimated because of the high diversity of fish and the small number of these studies on this community. This emphasizes the importance of the present survey for deepening knowledge about the local fauna.

\section{Acknowledgements}

We are grateful to Rodrigo Junio da Graça, Guilherme Pomaro Casali, Eloiza M. Capparros, Flávia Sicielli de Lima, Ana Paula C. L. Pizani, Carolina L. Evangelista, Fábio H. Yamada, Fabrício H. Oda, Eliane S. Fernandes and Letícia C. Karling for providing data/repository of the Universidade Estadual de Maringá. We thank Jaime L. Pereira for the map design. The staff of the Museu de Ictiologia at Universidade Estadual de Maringá, inspired us to do the work by updating the host list.

We also thank the Programa de Pós-Graduação em Ecologia de Ambientes Aquáticos Continentais (PEA), Programa de Pós-Graduação em Biologia Comparada (PGB), Núcleo de Pesquisas em Limnologia, Ictiologia e Aquicultura (NUPELIA), Pesquisas de Longa Duração (PELD-site 6), Conselho Nacional de Desenvolvimento Científico e Tecnológico (CNPq), Fundação Araucária and Coordenação de Aperfeiçoamento de Pessoal de Nível Superior (CAPES) for logistical support and scholarships.

\section{References}

Agostinho AA, Gomes LC, Santos NCL, Ortega JCG, Pelicice FM. Fish assemblages in Neotropical reservoirs: Colonization patterns, impacts and management. Fish Res 2016; 173(1): 26-36. http://dx.doi.org/10.1016/j.fishres.2015.04.006.

Agostinho AA, Gomes LC, Zalewski M. The importance of floodplains for the dynamics of fish communities of the upper river Paraná. Ecohydrol Hydrobiol 2001; 1(1-2): 209-217.

Agostinho AA, Júlio HFJr. Peixes da bacia do alto rio Paraná. In: Lowe-McConnel RH. Estudos ecológicos de comunidades de peixes tropicais. São Paulo: EDUSP; 1999. p. 374-400.

Ahyong ST, Lowry JK, Alonso M, Bamber RN, Boxshall GA, Castro P, et al. Subphylum Crustacea Brünnich, 1772. In: Zhang, Z.-Q. (Ed.) Animal biodiversity: An outline of higher-level classification and survey of taxonomic richness. Zootaxa 2011; 3148(1): 165-191. https:// doi.org/10.11646/zootaxa.3148.1.33

Aznar FJ, Balbuena JA, Fernández M, Raga JA. Living together: the parasites of marine mammals. In: Evans PGH, Raga JA, editors. Marine Mammals. New York: Kluwer Academic/Plenum Publishers; 2001. p. 385-423.

Brusca RC, Moore W, Shuster SM. Flagellata. In: Brusca RC, Moore W, Shuster SM. Invertebrados. 3rd ed. Rio de Janeiro: Guanabara Koogan; 2018a. p. 85-174.

Brusca RC, Moore W, Shuster SM. Myxozoa. In: Brusca RC, Moore W, Shuster SM. Invertebrados. 3rd ed. Rio de Janeiro: Guanabara Koogan; 2018b. p. 326-398. 
Brusca RC, Moore W, Shuster SM. Hirudinóideos. In: Brusca RC, Moore W, Shuster SM. Invertebrados. 3rd ed. Rio de Janeiro: Guanabara Koogan; 2018c. p. 649-724.

Brusca RC, Moore W, Shuster SM. Acari. In: Brusca RC, Moore W, Shuster SM. Invertebrados. 3rd ed. Rio de Janeiro: Guanabara Koogan; 2018d. p. 1081-1145.

Cohen SC, Justo MC, Kohn A. South American Monogenoidea parasites of fishes, amphibians and reptiles. Rio de Janeiro: Ed. Oficina de Livros; 2013.

Da Graça RJ, Fabrin TMC, Gasques LS, Prioli SMAP, BalbuenaJA, Prioli AJ, et al. Topological congruence between phylogenies of Anacanthorus spp. (Monogenea: Dactylogyridae) and their Characiformes (Actinopterygii) hosts: A case of host-parasite cospeciation. PLoS One 2018; 13(3): e0193408. http://dx.doi.org/10.1371/journal.pone.0193408. PMid:29538463.

Dos Reis CMR, Da Silva CN, Ueta MT, Da Silva JCJ, Ceccarelli PS, Alegrette SM. Evaluation of trace elements in Prochilodus lineatus (Valenciennes, 1837) (Characiformes, Prochilodontidae) from the Mogi Guaçu river infected for Acanthocephala Neoechynorhyncus curemai Noronha, 1973 (Acanthocephala). J Environ Anal Toxicol 2017; 7(03): 458. http://dx.doi.org/10.4172/2161-0525.1000458.

Eiras JC, Adriano EA. Myxozoa. In: Pavanelli GC, Takemoto RM, Eiras JC, editors Parasitologia de Peixes de água doce do Brasil. Maringá: EDUEM; 2013. p. 317-332.

Eiras JC, Takemoto RM, Pavanelli GC. Crustacea. In: Eiras JC, Takemoto RM, Pavanelli GC. Diversidade dos parasitas de peixes de água doce do Brasil. Maringá: Clichetec; 2010.

Eiras JC, Takemoto RM, Pavanelli GC, Adriano EA. About the biodiversity of parasites of freshwater fish from Brazil. Bull Eur Assoc Fish Pathol 2011; 31(4): 161.

Esch GW, Barger MA, Fellis KJ. The transmission of digenetic trematodes: style, elegance, complexity. Integr Comp Bio/ 2002; 42(2): 304312. http://dx.doi.org/10.1093/icb/42.2.304. PMid:21708722.

Ferrari-Hoeinghaus AP, Bellay S, Takemoto RM, Pavanelli GC. A new species of Demidospermus Suriano, 1983 (Monogenea, Dactylogyridae) parasitic on Loricariichthys platymetopon Isbrücker et Nijssen (Loricariidae, Siluriformes) from the Upper Paraná River floodplain, Brazil. Acta Parasitol 2010; 55(1): 16-19. http://dx.doi.org/10.2478/s11686-010-0007-5.

Franceschini L, Zago AC, Müller MI, Francisco CJ, Takemoto RM, Da Silva RJ. Morphology and molecular characterization of Demidospermus spirophallus n. sp., D. prolixus n. sp. (Monogenea: Dactylogyridae) and a redescription of $D$. anus in siluriform catfish from Brazil.J Helminthol 2018; 92(2): 228-243. http://dx.doi.org/10.1017/S0022149X17000256. PMid:28382887.

Gasques LS, Graça RJ, Prioli SMAP, Takemoto RM, Prioli AJ. Molecular characterization of Urocleidoides cuiabai and U. malabaricusi (Monogenea: Dactylogyridae) from the trahira fish Hoplias aff. malabaricus in the Paraná River, Brazil. J Helminthol 2016; 90(6): 693-697. http://dx.doi.org/10.1017/S0022149X15000966. PMid:26603609.

Gardner SL, Campbell ML. Parasites as probes for biodiversity. J Parasito/ 1992; 78(4): 596-600. http://dx.doi.org/10.2307/3283534. PMid:1635018.

Gois KS, Pelicice FM, Gomes LC, Agostinho AA. Invasion of an Amazonian cichlid in the upper Paraná River: facilitation by dams and decline of a phylogenetically related species. Hydrobiologia 2015; 746(1): 401-413. http://dx.doi.org/10.1007/s10750-014-2061-8.

Horne AJ, Goldman CR. Limnology. New York: McGraw-Hill; 1994.

Junk WJ, Bayley PB, Sparks RE. The flood pulse concept in river-floodplain systems. In: Dodge DP, editor. Proceedings of the International Large River Symposium (LARS). Toronto: Ontario Ministry of Natural Resources; 1989. (Canadian Special Publication of Fisheries and Aquatic Sciences; 106).

Karling LC, Bellay S, Takemoto RM, Pavanelli GC. A new species of Jainus (Monogenea), gill parasite of Schizodon borellii (Characiformes, Anostomidae) from the upper Paraná river floodplain, Brazil. Acta Sci Bio/Sci 2011; 33(2): 227-231. http://dx.doi.org/10.4025/actascibiolsci. v33i2.6168.

Karling LC, Lopes LPC, Takemoto RM, Pavanelli GC. New species of Tereancistrum (Dactylogyridae) monogenean parasites of Schizodon borellii (Characiformes, Anostomidae) from Brazil, and emended diagnosis for T. parvus. Acta Sci Biol Sci 2014; 36(3): 365-369. http://dx.doi. org/10.4025/actascibiolsci.v36i3.20216.

Khalil FL, Jones A, Bray AR. Keys to the Cestode Parasites of Vertebrates. Cambridge: Cab International, University Press; 1994.

Kohn A, Fernandes BM, Cohen SC. South American trematodes parasites of fishes. Rio de Janeiro: Imprinta Express Ltda; 2007.

LandsbergJH, Blakesley BA, Reese RO, McRae G, Forstchen PR. Parasites of fish as indicators of environmental stress. Environ Monit Assess 1998; 51(1-2): 211-232. http://dx.doi.org/10.1023/A:1005991420265.

Locke SA, Marcogliese DJ, Valtonen ET. Vulnerability and diet breadth predict larval and adult parasite diversity in fish of the Bothnian Bay. Oecologia 2014; 174(1): 253-262. http://dx.doi.org/10.1007/s00442-013-2757-x. PMid:24026499.

Luque JL, Poulin R. Linking ecology with parasite diversity in Neotropical fishes.J Fish Bio/ 2008; 72(1): 189-204. http://dx.doi.org/10.1111/ j.1095-8649.2007.01695.x. 
Luque JL, Vieira FM, Takemoto RM, Pavanelli GC, Eiras JC. Checklist of Crustacea parasitizing fishes from Brazil. Check List 2013; 9(6): 14491470. http://dx.doi.org/10.15560/9.6.1449.

Luque JL. Biologia, epidemiologia e controle de parasitos de peixes. Rev Bras Parasitol Vet 2004; 13(Suppl 1): 161-165.

Marchiori NC, Martins ML. Flagellata. In: Pavanelli GC, Takemoto RM, Eiras JC, editors. Parasitologia de Peixes de água doce do Brasil. Maringá; EDUEM; 2013. p. 201-215.

Moravec F. Nematodes of freshwater fishes of the neotropical region. Czech Republic: Academia Praha, Academy of Sciences of the Czech Republic; 1998.

Nachev M, Sures B. Seasonal profile of metal accumulation in the acanthocephalan Pomphorhynchus laevis: a valuable tool to study infection dynamics and implications for metal monitoring. Parasit Vectors 2016; 9(1): 300. http://dx.doi.org/10.1186/s13071-016-1576-4. PMid:27216617.

Ota RR, Deprá GC, da Graça WJ, Pavanelli CS. Peixes da planície de inundação do alto rio Paraná e áreas adjacentes: revised, annotated and updated. Neotrop Ichthyo/ 2018; 16(2): e170094. http://dx.doi.org/10.1590/1982-0224-20170094.

Paller VGV, Resurreccion DJB, De La Cruz CPP, Bandal MZ Jr. Acanthocephalan parasites (Acanthogyrus sp.) of Nile tilapia (Oreochromis niloticus) as biosink of lead (Pb) contamination in a Philippine freshwater lake. Bull Environ Contam Toxicol 2016; 96(6): 810-815. http:// dx.doi.org/10.1007/s00128-016-1790-y. PMid:27052033.

Pavanelli GC, Machado MH, Takemoto RM. Fauna helmíntica de peixes do rio Paraná, região do Porto Rico, Paraná. In: Vazzoler AEAM, Agostinho AA, Hahn NS, editors. A planície de inundação do alto rio Paraná: aspectos físicos, biológicos e socioeconômico. Maringá: EDUEM; 1997. p. 307-342.

Pavanelli GC, Pizani APCL, Mendes PB. Cestoda. In: Pavanelli GC, Takemoto RM, Eiras JC, editors. Parasitologia de Peixes de água doce do Brasil. Maringá: EDUEM; 2013. p. 317-332.

Poulin R. The evolution of monogenean diversity. Int J Parasitol 2002; 32(3): 245-254. http://dx.doi.org/10.1016/S0020-7519(01)00329-0. PMid:11835968.

Raga JA, Fernández M, Balbuena JA, Aznar J. Parasites. In: Perrin, WF, Würsig B, Thewissen JGM, editors. Encyclo Mar Mammals. San Diego: Academic Press; 2008. p. 821-830.

Richardson DJ, Moser WE, Hammond Cl, Lazo-Wasem EA. New host and geographic distribution records for Glossiphoniid leeches associated with turtles in Southern New England. Comp Parasitol 2015; 82(2): 240-243. http://dx.doi.org/10.1654/4737.1.

Sures B, Nachev M, Selbach C, Marcogliese DJ. Parasite responses to pollution: what we know and where we go in 'Environmental Parasitology'. Parasit Vectors 2017; 10(1): 65. http://dx.doi.org/10.1186/s13071-017-2001-3. PMid:28166838.

Takemoto RM, Pavanelli GC, Lizama MAP, Lacerda ACF, Yamada FH, Moreira LHA, et al. Diversity of parasites of fish from the Upper Paraná River floodplain, Brazil. Braz J Biol 2009;69(2 Suppl): 691-705. http://dx.doi.org/10.1590/S1519-69842009000300023. PMid:19738975.

Taraschewski H. Host-parasite interactions in Acanthocephala: A morphological approach. Adv Parasitol 2000; 46: 1-179. http://dx.doi. org/10.1016/S0065-308X(00)46008-2. PMid:10761555.

Tavernari FC, Takemoto RM, Lacerda ACF, Pavanelli GC. A new species of Demidospermus Suriano, 1983 (Monogenea) parasite of gills of Auchenipterus osteomystax (Auchenipteridae), from the upper Paraná river floodplain, Brazil. Acta Sci Bio/ Sci 2010; 32(1): 79-81. http:// dx.doi.org/10.4025/actascibiolsci.v32i1.3368.

Thatcher VE. Trematódeos neotropicais. Manaus: Instituto Nacional de Pesquisas da Amazônia; 1993.

Thatcher VE. Aquatic biodiversity in Latin America. Vol 1. Amazon fish parasites. Sofia: Pensoft; 2006.

Thomaz SM, Bini LM, Bozelli RL. Floods increase similarity among aquatic habitats in river-floodplain systems. Hydrobiologia 2007; 579(1): 1-13. http://dx.doi.org/10.1007/s10750-006-0285-y.

Thomaz SM, Pagioro TA, Bini LM, Roberto MC, Rocha RRA. Limnological characterization of the aquatic environments and the influence of hydrometric levels. In: Thomaz SM, Agostinho AA, Hahn NS, editors. The Upper Paraná river and its Floodplain: Physical Aspects, Ecology and Conservation. Leiden, The Netherlands: Backuys Publishers; 2004. p. 75-102

Toussaint A, Charpin N, Brosse S, Villéger S. Global functional diversity of freshwater fish is concentrated in the Neotropics while functional vulnerability is widespread. Sci Rep 2016; 6(1): 22125. http://dx.doi.org/10.1038/srep22125. PMid:26980070.

Vidal-Martínez VM. Metazoan parasites in the neotropics: A systematic and ecological perspective. México: UNAM; 2000.

Vitule JR, Agostinho AA, Azevedo-Santos VM, Daga VS, Darwall WRT, Fitzgerald DB, et al. We need better understanding about functional diversity and vulnerability of tropical freshwater fishes. Biodivers Conserv2017; 26(3): 757-762. http://dx.doi.org/10.1007/s10531-016-1258-8.

Yamada FH, Moreira LHA, Ceschini TL, Takemoto RM, Pavanelli GC. Novas ocorrências de metacercária de Austrodiplostomum compactum (Lutz, 1928) (Platyhelminthes: Digenea) parasito de olhos de peixes da bacia do rio Paraná. Rev Bras Parasitol Vet 2008; $17(3): 163-166$. http://dx.doi.org/10.1590/S1984-29612008000300010. PMid:19245765.

Yamaguti S. Systema Helminthum: The cestodes of vertebrates. New York: Interscience Publishers INC; 1959. (vol. 2). 
Parasites in fish from the Paraná River

\section{Supplementary Material}

Supplementary material accompanies this paper.

Legenda S1. Supplementary Material: Articles.

This material is available as part of the online article from http://www.scielo.br/RBPV 